\title{
DIFFERENTIATING THE ABSOLUTELY CONTINUOUS INVARIANT MEASURE OF AN INTERVAL MAP $f$ WITH RESPECT TO $f$.
}

by David Ruelle*.

Abstract. Let the map $f:[-1,1] \rightarrow[-1,1]$ have a.c.i.m. $\rho$ (absolutely continuous $f$-invariant measure with respect to Lebesgue). Let $\delta \rho$ be the change of $\rho$ corresponding to a perturbation $X=\delta f \circ f^{-1}$ of $f$. Formally we have, for differentiable A,

$$
\delta \rho(A)=\sum_{n=0}^{\infty} \int \rho(d x) X(x) \frac{d}{d x} A\left(f^{n} x\right)
$$

but this expression does not converge in general. For $f$ realanalytic and Markovian in the sense of covering $(-1,1) m$ times, and assuming an analytic expanding condition, we show that

$$
\lambda \mapsto \Psi(\lambda)=\sum_{n=0}^{\infty} \lambda^{n} \int \rho(d x) X(x) \frac{d}{d x} A\left(f^{n} x\right)
$$

is meromorphic in $\mathbf{C}$, and has no pole at $\lambda=1$. We can thus formally write $\delta \rho(A)=\Psi(1)$.

\footnotetext{
* Mathematics Dept., Rutgers University, and IHES. 91440 Bures sur Yvette, France.
} $<$ ruelle@ihes.fr $>$ 
We postpone a discussion of the significance of our result, and start to describe the conditions under which we prove it. Note that these conditions are certainly too strong: suitable differentiability should replace analyticity, and a weaker Markov property should be sufficient. But the point of the present note is to show how it is that $\Psi(\lambda)$ has no pole at $\lambda=1$, rather than deriving a very general theorem.

\section{Setup.}

We assume that $f:[-1,1] \rightarrow[-1,1]$ is real analytic and piecewise monotone on $[-1,1]$ in the following sense: there are points $c_{j}(j=0, \ldots, m$, with $m \geq 2)$ such that $-1=c_{0}<c_{1}<\ldots<c_{m-1}<c_{m}=1$ and, for $j=0, \ldots, m$,

$$
f\left(c_{j}\right)=(-1)^{j+1}
$$

We assume that on $[-1,1]$ the derivative $f^{\prime}$ vanishes only on $Z=\left\{c_{1}, \ldots, c_{m-1}\right\}$, and that $f^{\prime \prime}$ does not vanish on $Z$. For $j=1, \ldots, m$, we have $f\left[c_{j-1}, c_{j}\right]=[-1,1]$. In particular, $f$ is Markovian. We shall also assume that $f$ is analytically expanding in the sense of Assumption A below. The purpose of this note is to prove the following:

Theorem. Under the above conditions, and Assumption A stated later, there is a unique $f$-invariant probability measure $\rho$ absolutely continuous with respect to Lebesgue on $[-1,1]$. If $X$ is real-analytic on $[-1,1]$, and $A \in \mathcal{C}^{1}[-1,1]$, then

$$
\Psi(\lambda)=\sum_{n=0}^{\infty} \lambda^{n} \int_{-1}^{1} \rho(d x) X(x) \frac{d}{d x} A\left(f^{n} x\right)
$$

extends to a meromorphic function in $\mathbf{C}$, without pole at $\lambda=1$.

Our proof depends on a change of variable which we now explain. We choose a holomorphic function $\omega$ from a small open neighborhood $U_{0}$ of $[-1,1]$ in $\mathbf{C}$ to a small open neighborhood $W$ of $[-1,1]$ in a Riemann surface which is 2 -sheeted over $\mathbf{C}$ near -1 and 1. We call $\varpi=\omega^{-1}: W \rightarrow U_{0}$ the inverse of $\omega$. We assume that $\omega(-x)=-\omega(x)$, $\omega( \pm 1)= \pm 1, \omega[-1,1]=[-1,1], \omega^{\prime}( \pm 1)=\omega^{\prime \prime \prime}( \pm 1)=0$. We have thus

$$
\omega( \pm(1-\xi))= \pm\left(1-C \xi^{2}+D \xi^{4} \ldots\right)
$$

with $C>0$ and, if $a>0$,

$$
\varpi\left( \pm\left(1-a \xi^{2}+b \xi^{3} \ldots\right)\right)= \pm\left(1-\sqrt{\frac{a}{C}} \xi+\frac{b}{2 \sqrt{a C}} \xi^{2} \ldots\right)
$$

[We may for instance take

$$
\omega(x)=\sin \frac{\pi x}{2} \quad, \quad \varpi(x)=\frac{2}{\pi} \arcsin x
$$

or

$$
\omega(x)=\frac{1}{16}\left(25 x-10 x^{3}+x^{5}\right) \quad, \quad \varpi(x)=\frac{16}{25} x \ldots
$$


The function $g: \varpi \circ f \circ \omega$ from $[-1,1]$ to $[-1,1]$ has monotone restrictions to the intervals $\varpi\left[c_{j-1}, c_{j}\right]=\left[d_{j-1}, d_{j}\right]$. It is readily seen that $g_{j}$ extends to a holomorphic function in a neighborhood of $\left[d_{j-1}, d_{j}\right]$, and that

$$
\begin{gathered}
g_{1}(-1+\xi)=-1+\sqrt{f^{\prime}(-1)} \xi+\alpha_{-} \xi^{3} \ldots \\
g_{m}(1-\xi)=(-1)^{m+1}\left(1-\sqrt{\left|f^{\prime}(1)\right|} \xi-\alpha_{+} \xi^{3} \ldots\right)
\end{gathered}
$$

with no $\xi^{2}$ terms in the right-hand sides [this follows from our choice of $\omega$, which has no $\xi^{3}$ term]. One also finds that, for $j=1, \ldots, m-1$

$$
\begin{gathered}
g_{j}\left(d_{j}-\xi\right)=(-1)^{j+1}\left(1-\sqrt{\frac{\left|f^{\prime \prime}\left(c_{j}\right)\right|}{2 C}} \omega^{\prime}\left(d_{j}\right) \xi+\gamma_{j} \xi^{2} \ldots\right) \\
g_{j+1}\left(d_{j}+\xi\right)=(-1)^{j+1}\left(1-\sqrt{\frac{\left|f^{\prime \prime}\left(c_{j}\right)\right|}{2 C}} \omega^{\prime}\left(d_{j}\right) \xi-\gamma_{j} \xi^{2} \ldots\right)
\end{gathered}
$$

where $\gamma_{j}$ is the same in the two relations. We note the following easy consequences of the above developments:

Lemma 1. Let $\psi_{j}:[-1,1] \rightarrow\left[d_{j-1}, d_{j}\right]$ be the inverse of $g_{j}$ for $j=1, \ldots, m$ (increasing for $j$ odd, decreasing for $j$ even). Then

$$
\begin{gathered}
\psi_{1}(-1+\xi)=-1+\frac{1}{\sqrt{f^{\prime}(-1)}} \xi+\beta_{-} \xi^{3} \\
\psi_{m}\left((-1)^{m+1}(1-\xi)\right)=1-\frac{1}{\sqrt{\left|f^{\prime}(1)\right|}} \xi+\beta_{+} \xi^{3}
\end{gathered}
$$

(there are no $\xi^{2}$ terms in the right-hand sides). If $j<m$,

$$
\begin{gathered}
\psi_{j}\left((-1)^{j+1}(1-\xi)\right)=d_{j}-\sqrt{\frac{2 C}{\left|f^{\prime \prime}\left(c_{j}\right)\right|}} \frac{1}{\omega^{\prime}\left(d_{j}\right)} \xi+\delta_{j} \xi^{2} \\
\psi_{j+1}\left((-1)^{j+1}(1-\xi)\right)=d_{j}+\sqrt{\frac{2 C}{\left|f^{\prime \prime}\left(c_{j}\right)\right|}} \frac{1}{\omega^{\prime}\left(d_{j}\right)} \xi+\delta_{j} \xi^{2}
\end{gathered}
$$

(with the same coefficient $\delta_{j}$ ).

As inverses of the $g_{j}$, the functions $\psi_{j}$ extend to holomorphic functions on a neighborhood of $[-1,1]$. We impose now the condition that $f$ is analytically expanding in the following sense:

Assumption A We have $[-1,1] \subset U \subset \mathbf{C}$, with $U$ bounded open connected, such that the $\psi_{j}$ extend to continuous functions $\bar{U} \mapsto \mathbf{C}$, holomorphic in $U$, and with $\psi_{j} \bar{U} \subset U$. $[\bar{U}$ denotes the closure of $U]$. 
Let $\phi$ be holomorphic on a neighborhood of $\bar{U}$. Given a sequence $\mathbf{j}=\left(j_{1}, \ldots, j_{\ell}, \ldots\right)$ we define $\phi_{\mathbf{j} \ell}=\phi \circ \psi_{j_{1}} \cdots \circ \psi_{j_{\ell}}$ and note that the $\phi_{\mathbf{j} \ell}$ are uniformly bounded in a neighborhood of $\bar{U}$. We may thus choose $\ell(r)$ for $r=1,2 \ldots$ such that the subsequence $\left(\phi_{\mathbf{j} \ell(r)}\right)_{r=1}^{\infty}$ converges uniformly on $\bar{U}$ to a limit $\tilde{\phi}_{\mathbf{j}}$. Writing $\tilde{U}=\cup_{j=1}^{m} \psi_{j} \bar{U}$ we have

$$
\max _{z \in \bar{U}}\left|\phi_{\mathbf{j} \ell(r)}\right| \geq \max _{z \in \tilde{U}}\left|\phi_{\mathbf{j} \ell(r)}\right| \geq \max _{z \in \bar{U}}\left|\phi_{\mathbf{j} \ell(r+1)}\right|
$$

so that $\max _{z \in \bar{U}}\left|\tilde{\phi}_{\mathbf{j}}\right|=\max _{z \in \tilde{U}}\left|\tilde{\phi}_{\mathbf{j}}\right|$ and, since $\tilde{U}$ is compact $\subset U$ connected, $\tilde{\phi}_{\mathbf{j}}$ is constant. Therefore $\phi$ is constant on $\cap_{\ell=0}^{\infty} \psi_{j_{1}} \circ \cdots \circ \psi_{j_{\ell}} \bar{U}$. Since this is true for all $\phi$, the intersection $\cap_{\ell=0}^{\infty} \psi_{j_{1}} \circ \cdots \circ \psi_{j_{\ell}} \bar{U}$ consists of a single point $\tilde{z}(\mathbf{j})$. Given $\epsilon>0$ we can thus, for each $\mathbf{j}$, find $\ell$ such that diam $\psi_{j_{1}} \circ \cdots \circ \psi_{j_{\ell}} \bar{U}<\epsilon$. Hence (using the compactness of the Cantor set of sequences $\mathbf{j}$ ) one can choose $L$ so that the $m^{L}$ sets

$$
\psi_{j_{1}} \circ \cdots \psi_{j_{L}} \bar{U}
$$

have diameter $<\epsilon$. The open connected set

$$
V=\cup_{j_{1}, \ldots, j_{L}} \psi_{j_{1}} \circ \cdots \psi_{j_{L}} U
$$

satisfies $[-1,1] \subset V \subset U$, and $\psi_{j} \bar{V}=\cup_{j_{1}, \ldots, j_{L}} \psi_{j} \circ \psi_{j_{1}} \circ \cdots \circ \psi_{j_{L}} \bar{U} \subset \cup_{j_{0}, j_{1}, \ldots, j_{L-1}} \psi_{j_{0}} \circ \psi_{j_{1}} \circ$ $\psi_{i_{L-1}} U=V$. This shows that $U$ can be replaced in Assumption A by a set $V$ contained in an $\epsilon$-neighborhood of $[-1,1]$.

Since we have shown above that $\operatorname{diam} \psi_{j_{1}} \circ \cdots \psi_{j_{L}} \bar{U}<\epsilon$, we see that $\psi_{1}^{L}$ maps a small circle around -1 strictly inside itself. We have thus $\psi_{1}^{\prime}(-1)<1$ (i.e., $f^{\prime}(-1)>1$ ) and similarly, if $m$ is odd, $\psi_{m}^{\prime}(1)<1$ (i.e., $\left.f^{\prime}(1)>1\right)$.

The following two lemmas state some easy facts to be used later.

Lemma 2. Let $H$ be the Hilbert space of functions $\bar{U} \rightarrow \mathbf{C}$ which are square integrable (with respect to Lebesgue) and holomorphic in $U$. The operator $\mathcal{L}$ on $H$ defined by

$$
(\mathcal{L} \Phi)(z)=\sum_{j=1}^{m}(-1)^{j+1} \psi_{j}^{\prime}(z) \Phi\left(\psi_{j}(z)\right)
$$

is holomorphy improving. In particular $\mathcal{L}$ is compact and trace-class.

Lemma 3. On $[-1,1]$ we have

$$
(\mathcal{L} \Phi)(x)=\sum_{j}\left|\psi_{j}^{\prime}(x)\right| \Phi\left(\psi_{j}(x)\right)
$$

hence $\Phi \geq 0$ implies $\mathcal{L} \Phi \geq 0$ ( $\mathcal{L}$ preserves positivity) and

$$
\int_{-1}^{1} d x(\mathcal{L} \Phi)(x)=\int_{-1}^{1} d x \Phi(x)
$$


( $\mathcal{L}$ preserves total mass).

Lemma 4. $\mathcal{L}$ has a simple eigenvalue $\mu_{0}=1$ corresponding to an eigenfunction $\sigma_{0}>0$. The other eigenvalues $\mu_{k}(k \geq 1)$ satisfy $\left|\mu_{k}\right|<1$, and their (generalized) eigenfunctions $\sigma_{k}$ satisfy $\int_{-1}^{1} d x \sigma_{k}(x)=0$.

Let $\left(\mu_{k}, \sigma_{k}\right)$ be a listing of the eigenvalues and generalized eigenfunctions of the traceclass operator $\mathcal{L}$. For each $\mu_{k}$ there is some $\sigma_{k}$ such that $\mathcal{L} \sigma_{k}=\mu_{k} \sigma_{k}$, hence

$$
\begin{gathered}
\left|\mu_{k}\right| \int_{-1}^{1} d x\left|\sigma_{k}(x)\right|=\int_{-1}^{1} d x\left|\mu_{k} \sigma_{k}(x)\right|=\int_{-1}^{1} d x\left|\left(\mathcal{L} \sigma_{k}\right)(x)\right| \\
\leq \int_{-1}^{1} d x\left(\mathcal{L}\left|\sigma_{k}\right|\right)(x)=\int_{-1}^{1} d x\left|\sigma_{k}(x)\right|
\end{gathered}
$$

hence $\left|\mu_{k}\right| \leq 1$. Denote by $S_{<}$and $S_{1}$ the spectral spaces of $\mathcal{L}$ corresponding to eigenvalues $\mu_{k}$ with $\left|\mu_{k}\right|<1$, and $\left|\mu_{k}\right|=1$ respectively. If $\sigma_{k} \in S_{<}$then, for some $n \geq 1$,

$$
0=\int_{-1}^{1} d x\left(\left(\mathcal{L}-\mu_{k}\right)^{n} \sigma_{k}\right)(x)=\int_{-1}^{1} d x\left(1-\mu_{k}\right)^{n} \sigma_{k}(x)
$$

hence $\int_{-1}^{1} d x \sigma_{k}(x)=0$.

On the finite dimensional space $S_{1}$, there is a basis of eigenvectors $\sigma_{k}$ diagonalizing $\mathcal{L}$ (if $\mathcal{L} \mid S_{1}$ had non-diagonal normal form, || $\mathcal{L}^{n}\left|S_{1}\right| \mid$ would tend to infinity with $n$, in contradiction with $\left.\int_{-1}^{1} d x\left|\left(\mathcal{L}^{n} \Phi\right)(x)\right| \leq \int_{-1}^{1} d x|\Phi(x)|\right)$. We shall now show that, up to multiplication by a constant $\neq 0$, we may assume $\sigma_{k} \geq 0$. If not, because $\sigma_{k}$ is continuous and the intervals $\psi_{j_{1}} \circ \cdots \circ \psi_{j_{n}}[-1,1]$ are small for large $n$ (mixing), we would have $\left|\left(\mathcal{L}^{n} \sigma_{k}\right)(x)\right|<\left(\mathcal{L}^{n}\left|\sigma_{k}\right|\right)(x)$ for some $n$ and $x$. This would imply $\int_{-1}^{1} d x\left|\left(\mathcal{L}^{n} \sigma_{k}\right)(x)\right|<$ $\int_{-1}^{1} d x\left|\sigma_{k}(x)\right|$ in contradiction with $\mathcal{L} \sigma_{k}=\mu_{k} \sigma_{k}$ and $\left|\mu_{k}\right|=1$. From $\sigma_{k} \geq 0$ we get $\mu_{k}=1$, and the corresponding eigenspace is at most one dimensional (otherwise it would contain functions not $\geq 0$ ). But we have $1 \notin S_{<}$because $\int_{-1}^{1} d x, 1 \neq 0$, so that $S_{1} \neq\{0\}$. Thus $S_{1}$ is spanned by an eigenfunction, which we call $\sigma_{0}$, to the eigenvalue $\mu_{0}=1$. Finally, $\sigma_{0}>0$ because if $\sigma_{0}(x)=0$ we would have also $\sigma_{0}(y)=0$ whenever $g^{n}(y)=x$, which is not compatible with $\sigma_{0}$ continuous $\neq 0$.

Lemma 5. If we normalize $\sigma_{0}$ by $\int_{-1}^{1} d x \sigma_{0}(x)=1$, then $\sigma_{0}(d x)=\sigma_{0}(x) d x$ is the unique $g$-invariant probability measure absolutely continuous with respect to Lebesgue on $[-1,1]$. In particular, $\sigma_{0}(d x)$ is ergodic.

For continuous $A$ on $[-1,1]$ we have

$$
\int_{-1}^{1} \sigma_{0}(d x)(A \circ g)(x)=\int_{-1}^{1} d x \sigma_{0}(x) A(g(x))=\int_{-1}^{1} d x\left(\mathcal{L} \sigma_{0}\right)(x) A(x)=\int_{-1}^{1} \sigma_{0}(d x) A(x)
$$

so that $\sigma_{0}(d x)$ is $g$-invariant. Let $\tilde{\sigma}(x) d x$ be another $g$-invariant probability measure absolutely invariant with respect to Lebesgue. Then, if $\tilde{\sigma} \neq \sigma_{0}$

$$
\int_{-1}^{1} d x\left|\sigma_{0}(x)-\tilde{\sigma}(x)\right|=\int_{-1}^{1} d x\left|\left(\mathcal{L}\left(\sigma_{0}-\tilde{\sigma}\right)\right)(x)\right|
$$




$$
<\int_{-1}^{1} d x\left(\mathcal{L}\left|\sigma_{0}-\tilde{\sigma}\right|\right)(x)=\int_{-1}^{1} d x\left|\sigma_{0}(x)-\tilde{\sigma}(x)\right|
$$

by mixing: contradiction.

Lemma 6. Let $H_{1} \subset H$ consist of those functions $\Phi$ with derivatives vanishing at \pm 1 : $\Phi^{\prime}(-1)=\Phi^{\prime}(1)=0$. Then $\mathcal{L} H_{1} \subset H_{1}$ and $\sigma_{0} \in H_{1}$.

$\mathcal{L} H_{1} \subset H_{1}$ is an easy calculation using Lemma 1. Furthermore, by Lemma $4, \sigma_{0}=$ $\lim _{n \rightarrow \infty} \mathcal{L}^{n} \frac{1}{2}$, and $\frac{1}{2} \in H_{1}$ implies $\sigma_{0} \in H_{1}$.

The image $\rho(d x)=\rho(x) d x$ of $\sigma_{0}(x) d x$ by $\omega$ is the unique $f$-invariant probability measure absolutely continuous with respect to Lebesgue on $[-1,1]$. We have

$$
\rho(x)=\sigma_{0}(\varpi x) \varpi^{\prime}(x)
$$

Consider now the expression

$$
\Psi(\lambda)=\sum_{n=0}^{\infty} \lambda^{n} \int_{-1}^{1} \rho(d x) X(x) \frac{d}{d x} A\left(f^{n} x\right)
$$

where we assume that $X$ extends to a holomorphic function in a neighborhood of $[-1,1]$ and $A \in \mathcal{C}^{1}[-1,1]$. For sufficiently small $|\lambda|$, the series defining $\Psi(\lambda)$ converges. Writing $B=A \circ \omega$ and $x=\omega y$ we have

$$
X(x) \frac{d}{d x} A\left(f^{n} x\right)=X(\omega y) \frac{1}{\omega^{\prime}(y)} \frac{d}{d y} B\left(g^{n} y\right)
$$

hence

$$
\Psi(\lambda)=\sum_{n=0}^{\infty} \lambda^{n} \int_{-1}^{1} d y \sigma_{0}(y) \frac{X(\omega y)}{\omega^{\prime}(y)} \frac{d}{d y} B\left(g^{n} y\right)
$$

Defining $Y(y)=\sigma_{0}(y) X(\omega y) / \omega^{\prime}(y)$, we see that $Y$ extends to a function holomorphic in a neighborhood of $[-1,1]$, which we may take to be $U$, except for simple poles at -1 and 1 . We may write

$$
\begin{gathered}
\int_{-1}^{1} d y \sigma_{0}(y) \frac{X(\omega y)}{\omega^{\prime}(y)} \frac{d}{d y} B\left(g^{n} y\right)=\int_{-1}^{1} d y Y(y) g^{\prime}(y) \cdots g^{\prime}\left(g^{n-1} y\right) B^{\prime}\left(g^{n} y\right) \\
=\int_{-1}^{1} d s\left(\mathcal{L}_{0}^{n} Y\right)(s) B^{\prime}(s)
\end{gathered}
$$

where

$$
\left(\mathcal{L}_{0} \Phi\right)(s)=\sum_{j=1}^{m}(-1)^{j+1} \Phi\left(\psi_{j} s\right)
$$

and we have thus

$$
\Psi(\lambda)=\sum_{n=0}^{\infty} \lambda^{n} \int_{-1}^{1} d s\left(\mathcal{L}_{0}^{n} Y\right)(s) B^{\prime}(s)
$$


Lemma 7. Let $H_{0} \subset H$ be the space of functions vanishing at -1 and 1 . Then $\mathcal{L}_{0} H_{0} \subset H_{0}$. This follows readily from Lemma 1 . [

Lemma 8. There are meromorphic functions $\Phi_{ \pm}$with Laurent series

$$
\Phi_{ \pm}(z)=\frac{1}{z \mp 1}+O(z \mp 1)
$$

at \pm 1 and $\Phi_{ \pm}(\mp 1)=0$ such that

$$
\begin{gathered}
\mathcal{L}_{0} \Phi_{-}=\sqrt{f^{\prime}(-1)} \Phi_{-} \\
\left\{\begin{array}{c}
\mathcal{L}_{0} \Phi_{+}=\sqrt{f^{\prime}(1)} \Phi_{+} \quad \text { if } m \text { is odd } \\
\mathcal{L}_{0}\left(\Phi_{+} / \sqrt{\left|f^{\prime}(1)\right|}+\Phi_{-} / \sqrt{f^{\prime}(-1)}\right)=\tilde{Y} \in H_{0} \quad \text { if } m \text { is even }
\end{array}\right.
\end{gathered}
$$

Define

$$
p_{ \pm}(z)=\frac{1}{z \mp 1}-\frac{1}{4}(z \mp 1)
$$

then Lemma 1 yields

$$
\begin{gathered}
\left(\mathcal{L}_{0}-\sqrt{f^{\prime}(-1)}\right) p_{-}=u_{-} \in H_{0} \\
\left\{\begin{array}{c}
\left(\mathcal{L}_{0}-\sqrt{f^{\prime}(1)}\right) p_{+}=u_{+} \in H_{0} \quad \text { if } m \text { is odd } \\
\mathcal{L}_{0} p_{+}+\sqrt{\left|f^{\prime}(1)\right|} p_{-}=u_{0} \in H_{0} \quad \text { if } m \text { is even }
\end{array}\right.
\end{gathered}
$$

Since $f^{\prime}(-1)>1$, Lemma 4 shows that $\mathcal{L}-\sqrt{f^{\prime}(-1)}$ is invertible on $H$, hence there is $v_{-}$ such that

$$
\left(\mathcal{L}-\sqrt{f^{\prime}(-1)}\right) v_{-}=u_{-}^{\prime}
$$

and since $\int_{-1}^{1} d x u_{-}^{\prime}(x)=0$, also $\int_{-1}^{1} d x v_{-}(x)=0$ and we can take $w_{-} \in H_{0}$ such that $w_{-}^{\prime}=v_{-}$. Then

$$
\left(\left(\mathcal{L}_{0}-\sqrt{f^{\prime}(-1)}\right) w_{-}\right)^{\prime}=\left(\mathcal{L}-\sqrt{f^{\prime}(-1)}\right) w_{-}^{\prime}=\left(\mathcal{L}-\sqrt{f^{\prime}(-1)}\right) v_{-}=u_{-}^{\prime}
$$

so that

$$
\left(\mathcal{L}_{0}-\sqrt{f^{\prime}(-1)}\right) w_{-}=u_{-}
$$

without additive constant because the left-hand side is in $H_{0}$ by Lemma 7 . In conclusion

$$
\left(\mathcal{L}_{0}-\sqrt{f^{\prime}(-1)}\right)\left(p_{-}-w_{-}\right)=0
$$

and we may take $\Phi_{-}=p_{-}-w_{-}$.

If $m$ is odd, $\Phi_{+}$is handled similarly. If $m$ is even, taking $\Phi_{+}=p_{+}$and writing $\tilde{Y}=u_{0} / \sqrt{\left|f^{\prime}(1)\right|}-w_{-}$we obtain

$$
\mathcal{L}_{0}\left(\frac{\Phi_{+}}{\sqrt{\left|f^{\prime}(1)\right|}}+\frac{\Phi_{-}}{\sqrt{f^{\prime}(-1)}}\right)=\tilde{Y} \in H_{0}
$$


which completes the proof.

We have $\sigma_{0} \in H_{1}$ (Lemma 6 ), and $X \circ \omega \in H_{1}$ by our choice of $\omega$. Also

$$
\omega^{\prime}( \pm(1-\xi))=2 C \xi-4 D \xi^{3} \ldots
$$

so that

$$
Y=\mathbf{C} \Phi_{-}+\mathbf{C} \Phi_{+}+H_{0}
$$

If $m$ is odd let $Y=c_{-} \Phi_{-}+c_{+} \Phi_{+}+Y_{0}$, with $Y_{0} \in H_{0}$. Then

$$
\Psi(\lambda)=\frac{c_{-}}{1-\lambda \sqrt{f^{\prime}(-1)}} \int_{-1}^{1} d s \Phi_{-}(s) B^{\prime}(s)+\frac{c_{+}}{1-\lambda \sqrt{f^{\prime}(1)}} \int_{-1}^{1} d s \Phi_{+}(s) B^{\prime}(s)+\Psi_{0}(\lambda)
$$

where $\Psi_{0}$ is obtained from $\Psi$ when $Y$ is replaced by $Y_{0}$.

If $m$ is even let $Y=c_{-} \Phi_{-}+\tilde{c}\left(\Phi_{+} / \sqrt{\left|f^{\prime}(1)\right|}+\Phi_{-} / \sqrt{f^{\prime}(-1)}\right)+Y_{0}$, with $Y_{0} \in H_{0}$. Then

$$
\begin{gathered}
\Psi(\lambda)=\frac{c_{-}}{1-\lambda \sqrt{f^{\prime}(-1)}} \int_{-1}^{1} d s \Phi_{-}(s) B^{\prime}(s)+\tilde{c} \int_{-1}^{1} d s\left(\frac{\Phi_{+}}{\sqrt{\left|f^{\prime}(1)\right|}}+\frac{\Phi_{-}}{\sqrt{f^{\prime}(-1)}}\right) B^{\prime}(s) \\
+\lambda \tilde{\Psi}(\lambda)+\Psi_{0}(\lambda)
\end{gathered}
$$

where $\tilde{\Psi}(\lambda)$ is obtained from $\Psi$ when $Y$ is replaced by $\tilde{Y}$.

Writing $\mu_{ \pm}=\sqrt{f^{\prime}( \pm 1)}$ we see that $\Psi(\lambda)$ has two poles at $\mu_{ \pm}^{-1}$ if $m$ is odd, and one pole at $\mu_{-}^{-1}$ if $m$ is even; the other poles are those of $\Psi_{0}(\lambda)$ and possibly $\tilde{\Psi}(\lambda)$. Since $Y_{0} \in H_{0}$ and $\mathcal{L}_{0} H_{0} \subset H_{0}$, we have

$$
\begin{gathered}
\Psi_{0}(\lambda)=\sum_{n=0}^{\infty} \lambda^{n} \int_{-1}^{1} d s\left(\mathcal{L}_{0}^{n} Y_{0}\right)(s) B^{\prime}(s)=-\sum_{n=0}^{\infty} \lambda^{n} \int_{-1}^{1} d s\left(\mathcal{L}_{0}^{n} Y_{0}\right)^{\prime}(s) B(s) \\
=-\sum_{n=0}^{\infty} \lambda^{n} \int_{-1}^{1} d s\left(\mathcal{L}^{n} Y_{0}^{\prime}\right)(s) B(s)
\end{gathered}
$$

It follows that $\Psi_{0}(\lambda)$ extends meromorphically to $\mathbf{C}$ with poles at the $\mu_{k}^{-1}$. We want to show that the residue of the pole at $\mu_{0}^{-1}=1$ vanishes. By Lemma $4, \int_{-1}^{1} d x \sigma_{k}(x)=0$ for $k \geq 1$. Thus, up to normalization, the coefficient of $\sigma_{0}$ in the expansion of $Y_{0}^{\prime}$ is

$$
\int_{-1}^{1} d x Y_{0}^{\prime}(x)=Y_{0}(1)-Y_{0}(-1)=0
$$

because $Y_{0} \in H_{0}$. Therefore $\Psi_{0}(z)$ is holomorphic at $z=1$, and the same argument applies to $\tilde{\Psi}(z)$, concluding the proof of the theorem.

\section{Discussion.}


It can be argued that the physical measure describing a physical dynamical system is an SRB (Sinai-Ruelle-Bowen) measure $\rho$ (see the recent reviews [11], [2] which contain a number of references), or an a.c.i.m. $\rho$ in the case of a map of the interval. But, typically, physical systems depend on parameters, and it is desirable to know how $\rho$ depends on the parameters (i.e., on the dynamical system). The dependence is smooth for uniformly hyperbolic dynamical systems (see [5], [6] and references given there), but discontinuous in general.

The present note is devoted to an example in support of an idea put forward in [8]: that derivatives of $\rho(A)$ with respect to parameters can be meaningfully defined in spite of discontinuities. An ambitious project would be to have Taylor expansions on a large set $\Sigma$ of parameter values and, using a theorem of Whitney [10], to connect these expansions by a function extrapolating $\rho(A)$ smoothly outside of $\Sigma$. In a different dynamical situation, that of KAM tori, a smooth extension à la Whitney has been achieved by Chierchia and Gallavotti [3], and Pöschel [4].

In our study we have considered only a rather special set $\Sigma$ consisting of maps satisfying a Markov property. (Reference [1] should be consulted for a discussion of the poles encountered in the study of a Markovian map $f$ ). Note that the studies of a.c.i.m. for maps of the interval, and of SRB measures for Hénon-like maps, are typically based on perturbations of a map satisfying a Markov property (for the use of slightly more general Misiurewicz-type maps see [9], which also gives references to earlier work).

The function $\Psi(\lambda)$ that we have encountered is related to the susceptibility $\omega \mapsto \Psi\left(e^{i \omega}\right)$ giving the response of a system to a periodic perturbation. The existence of a holomorphic extension of the susceptibility to the upper half complex plane is expected to follow from causality (causality says that cause preceeds effect, resulting in a response function $\kappa$ having support on the positive half real axis, and its Fourier transform $\hat{\kappa}$ extending holomorphically to the upper half complex plane). A discussion of nonequilibrium statistical mechanics [7] shows that the expected support and holomorphy properties hold close to equilibrium, or if uniform hyperbolicity holds. In the example discussed in this note, $\kappa$ has the right support property, but increases exponentially at infinity, and holomorphy in the upper half plane fails, corresponding the existence of a pole of $\Psi$ at $\lambda=1 / \sqrt{f^{\prime}(-1)}$. This might be expressed by saying that $\rho$ is not linearly stable. The physically interesting situation of large systems (thermodynamic limit) remains quite unclear at this point.

Acknowledgments. For many discussions on the subject of this note, I am indebted to V. Baladi, M. Benedicks, G. Gallavotti, M. Viana, and L.-S. Young.

\section{References.}

[1] V. Baladi, Y. Jiang, H.H. Rugh. "Dynamical determinants via dynamical conjugacies for postcritically finite polynomials." J. Statist. Phys. 108,973-993(2002).

[2] C. Bonatti, L. Diaz, and M. Viana. Dynamics beyond uniform hyperbolicity: a global geometric and probabilistic approach. Springer, to appear.

[3] L. Chierchia and G. Gallavotti. "Smooth prime integrals for quasi-integrable Hamiltonian systems." Nuovo Cim. 67B,277-295(1982).

[4] J. Pöschel. "Integrability of Hamiltonian systems on Cantor sets." Commun. in Pure and Applied Math. 35,653-696(1982). 
[5] D. Ruelle. "Differentiation of SRB states." Commun. Math. Phys. 187,227241(1997); "Correction and complements." Commun. Math. Phys. 234,185-190(2003).

[6] D. Ruelle. "Differentiation of SRB states for hyperbolic flows." In preparation.

[7] D. Ruelle. "Smooth dynamics and new theoretical ideas in nonequilibrium statistical mechanics." J. Statist. Phys. 95,393-468(1999).

[8] D. Ruelle. "Application of hyperbolic dynamics to physics: some problems and conjectures." Bull. Amer. Math. Soc. (N.S.) 41,275-278(2004).

[9] Q. Wang and L.-S. Young. "Towards a theory of rank one attractors." Preprint

[10] H. Whitney. "Analytic expansions of differentiable functions defined in closed sets." Trans. Amer. Math. Soc. 36,63-89(1934).

[11] L.-S. Young. "What are SRB measures, and which dynamical systems have them?" J. Statist. Phys. 108,733-754(2002). 